\title{
The Effects of Exercise on Serum Chemistry, Non-Esterified Fatty Acid, Insulin and Glucagon Dynamics during a 400 Meter Sprint in Racing Greyhounds
}

\author{
Michael J. Bell1, Emily M. Donaldson², Sabine Mann³, Robert L. Gillette4, Corri B. Levine², \\ Joseph J. Wakshlag ${ }^{*}$ \\ ${ }^{1}$ Craigieburn Animal Hospital, 9 Craigieburn Rd., Craigieburn, Australia \\ ${ }^{2}$ Cornell University College of Veterinary Medicine, Department of Clinical Sciences, Ithaca, NY, USA \\ ${ }^{3}$ Cornell University College of Veterinary Medicine, Department of Population Medicine, Ithaca, NY, USA \\ ${ }^{4}$ Red Bank Referral Center, Sports Medicine and Rehabilitation Service, Red Bank, NJ, USA \\ Email: Jw37@cornell.edu
}

Received 18 May 2015; accepted 26 June 2015; published 29 June 2015

Copyright (C) 2015 by authors and Scientific Research Publishing Inc.

This work is licensed under the Creative Commons Attribution International License (CC BY). http://creativecommons.org/licenses/by/4.0/

\section{(c) (i) Open Access}

\begin{abstract}
The effects of Greyhound racing on plasma insulin, glucagon and non-esterified fatty acid have not been extensively studied. We sought to examine the effects of exercise in 19 greyhounds trialed over 400 meters on serum biochemistry, osmolality, nonessential fatty acids, and the major glucoregulatory hormones insulin and glucagon, before and after racing. Due to the non-traditional dietary patterns of many racing Greyhounds, the diet was analyzed for macro and micro-nutrient assessment to determine adequacy of the diet. Many significant biochemical changes were observed and agreed with previous studies, except for low resting glucose, low post-exercise globulin and phosphorus concentrations, and a high CK both before and after exercise. Insulin concentrations increased 10 minutes post-exercise which might be a result of the rise in serum glucose observed. Non-esterified fatty acids decreased 10 minutes post-exercise, as a likely result of the insulin facilitated cellular uptake of fatty acids early in recovery. Glucagon concentrations did not change after exercise despite the rise in insulin. The diet analysis found some mineral deficiencies in sodium, selenium, iodine and copper; and contained a far higher protein and fat content, being atypical of diets that have been shown to improve the performance in racing Greyhounds.
\end{abstract}

\section{Keywords}

Greyhound, Insulin, Glucagon, Non-Esterified Fatty Acid, Glucose

\footnotetext{
${ }^{*}$ Corresponding author.
}

How to cite this paper: Bell, M.J., Donaldson, E.M., Mann, S., Gillette, R.L., Levine, C.B. and Wakshlag, J.J. (2015) The Effects of Exercise on Serum Chemistry, Non-Esterified Fatty Acid, Insulin and Glucagon Dynamics during a 400 Meter Sprint in Racing Greyhounds. Open Journal of Veterinary Medicine, 5, 142-151. http://dx.doi.org/10.4236/ojvm.2015.56020 


\section{Introduction}

Greyhound racing continues to be a popular sport in Australia, and portions of Europe, and the United States. The racing Greyhound has been a common sprinting dog prototype for evaluation of hemodynamic and intramuscular changes in relationship to exercise [1]-[8]. Commonly observed changes include significant shifts in acid-base balance and electrolyte concentrations [1]-[8]. Elevated osmolality and hematocrit are seen after racing in nearly every study [1]-[7]; however, these changes are transient and return to resting (or below resting) within 1 hour post-racing [5] [7]. The predominant theory is that intravascular fluid moves into the extravascular space during this strenuous exercise and leads to hemoconcentration [2]-[7]. However, it is possible that the increase in hematocrit is due to dehydration [5] [7] or splenic contraction [1] [2] [5] [8] [9].

To date, few studies have focused on glucose homeostasis and its relationship to sprinting dogs [10]. In general, dogs are considered as highly aerobic animals which has major implications for energy utilization during exercise [11]-[13]. In dogs, regulation of glucose production shifts from glucagon to catecholamines when performing high intensity exercise [10]. This change in glucose regulation is well documented in humans where a catecholamine spike occurs with a concomitant rise in insulin during intense exercise that dramatically declines during recovery [14].

The resting blood glucose observed in racing Greyhounds has been highly variable, between $60-100 \mathrm{mg} / \mathrm{dL}$ at rest, and the diet composition is often not reported [1] [5] [7] [8]. When examining the literature many studies performed by Hill and colleagues [4] utilized retired Greyhounds being fed commercial dog food, while the prior studies in the field do not report the dietary constituents or the nutrient composition of the diet fed during the trials, which may be influencing the resting glucose homeostasis [1]-[5] [7] [8]. This makes it difficult to fully understand the glucose dynamics observed across these studies. In humans as well as in dogs, hyperglycemia can occur after short intense bouts of exercise and has been observed in Greyhounds. Decreases in serum insulin have been observed in sled dogs and foxhounds immediately after exercise, yet whether or not insulin or glucagon changes occur in racing Greyhounds after sprinting has not been reported [2]-[5] [7] [14] [15].

Conversely, fat metabolism has not been thought to be a major component of energy regulation in racing Greyhounds. However, McClelland and colleagues studied the capability of dogs (breed unspecified) to utilize fatty acids during exercise of varying intensity [13]. They observed that blood fatty acid concentrations increase during periods of exercise in dogs, which was presumed to be due to increased binding of fatty acids to albumin during lipolysis. A single Greyhound study has shown a rise in free fatty acids which can be partially attributed to hemoconcentration as well as increased lipolysis [8]. Changes in non-esterified fatty acid (NEFA) concentrations have been reported to increase or decrease depending on duration of running, with sprinting activities showing some discrepancy regarding increases and decreases due to exercise [8] [13] [16].

The aim of our study was to examine the serum chemistry, NEFA, osmolality and the major glucoregulatory hormones (plasma insulin and glucagon) in competitive racing Greyhounds that are fed a typical non-commercial ration, similar to what is fed in many kennels (chicken, horse meat, bread and supplements).

\section{Materials and Methods}

\subsection{Greyhound Trial and Collections}

The exercise and sampling was approved by the Cornell University Institutional Animal Care and Use Committee. Nineteen Greyhounds from a single competitive kennel managed similarly to other regional kennels, were enrolled in the study with informed owner consent. All dogs were between the age of 16 and 20 months old with 11 being intact females and 8 being intact males undergoing conditioning for competitive Greyhound racing. The trial started at 8 am $\left(12^{\circ} \mathrm{C}\right)$ and all exercise was finished by 9:30 am $\left(12^{\circ} \mathrm{C}\right)$ with the last blood draws occurring by $11: 30 \mathrm{am}\left(14^{\circ} \mathrm{C}\right)$. Relative humidity during the trial was $95 \%$. All dogs were fasted before the trial with their past meal being $4 \mathrm{pm}$ the evening before the trial, and no dogs had been trained within 72 hours of the trial. Ten milliliters of whole blood was collected from each Greyhound at three time points: prior to the exercise bout in the kennel environment which was 200 meters away from the track with no visual contact with any activities on the track, 10 minutes post exercise at the track, and 120 minutes post exercise at the kennel. In all cases, the distance trialed was 400 meters on an oval dirt track chasing a lure individually. Body condition score on a 1 - 9 scale [17] was also assessed and recorded. All samples were collected from the cephalic vein and immediately placed in $10 \mathrm{ml}$ lithium heparin vacutainer blood tubes. They were then transported back to the clinic within 10 
minutes and centrifuged at $3800 \mathrm{~g}$ for 5 minutes and minimally 0.5 milliliters of separated plasma from each sample was placed into each of 3 plastic polypropylene collecting vials. Samples were then frozen at $-4^{\circ} \mathrm{C}$ until transportation approximately 1 week later on dry ice to the principle investigators laboratory where the samples were stored at $-80^{\circ} \mathrm{C}$ for approximately 1 week before analyses.

\subsection{Plasma Analyses}

Serum biochemical analytes (sodium, chloride, potassium, bicarbonate, calcium, phosphorus, magnesium, glucose, total protein, albumin, globulin, creatinine, urea, cholesterol, alanine aminotransferase [ALT], aspartate aminotransferase [AST], and creatine kinase [CK]) were analyzed at baseline, 10 minutes after exercise and 120 minutes after exercise using a Olympus AU5400 automated analyzer (Olympus America, Quakertown, PA). Plasma osmolality was assessed at baseline and 10 minutes after exercise using the Advanced 3250 Osmometer (Advanced Inc., Norwood, MA). Concentration of NEFA was analyzed for all samples in duplicate by colorimetric measurement of an enzymatic reaction according to the manufacturer's directions (HR Series NEFA-HR [2]; Wako Life Sciences, Mountain View, CA) with a microplate spectrophotometer (Epoch, Biotek, Winooski, VT). Intra- and inter-assay CV were 4.9 and 7.4\%, respectively; and all samples were run on three plates. Insulin and glucagon concentrations were measured using competitive radioimmunoassays at the certified New York State Diagnostic Laboratory at Cornell University (Porcine Insulin RIA, Millipore, Concord MA). All samples for insulin were run in two assays. Intra- and interassay coefficients of variation were $4.5 \%$ and $5.0 \%$, respectively. Glucagon was determined for all samples in duplicate using a radioimmunoassay kit according to manufacturer instruction (Glucagon RIA kit, Millipore, St. Charles, MO). In-house canine control samples were run every 28 samples. All samples were run in two assays. Intra- and interassay (CV) was determined as 3.9 and $4.9 \%$, respectively.

\subsection{Statistical Analyses}

Data was assessed for normalcy using a Shapiro Wilkes test, which revealed that a majority of the data was normally distributed except for NEFA, insulin and glucagon analyses. For the serum chemistry parameters, a one-way analysis of variance was performed with a Tukey's post hoc analysis to determine differences between resting (time 0), 10 minutes after exercise and 120 minutes after exercise. Lack of normality in NEFA, glucagon and insulin led to Friedman's analysis of variance with Tukey's post hoc analysis. Statistical significance was set at $\mathrm{p}<0.05$ for all testing.

\subsection{Diet Analyses}

A typical meal for one Greyhound was made and a sample of the diet was sent in for a basic macronutrient and mineral analysis through the Australian Government National Measures Institute. Substrates assessed were crude protein, crude fiber, crude fat, nitrogen free extract and total kilojoules. Minerals assessed were calcium, phosphorus, sodium, potassium, magnesium, iron, zinc, manganese, and copper. Further vitamin, selenium and iodine content of the data were assessed utilizing the United States Department of Agriculture Nutrient Database to complete the dietary analysis.

\section{Results}

\subsection{Plasma Electrolytes and Osmolality}

Plasma electrolytes exhibited a range of changes (Table 1). Plasma sodium showed a mild yet significant increase after exercise $(\mathrm{p}<0.01)$ and by 120 minutes after exercise the plasma sodium concentrations fell below the resting concentrations $(\mathrm{p}<0.01)$. Potassium concentrations in plasma were no different between resting and 10 minutes after exercise, but were decreased from baseline by 120 minutes after exercise $(p<0.01)$. Chloride showed a decrease 10 minutes after exercise when compared to baseline, but not 120 minutes after exercise (p < 0.01). Plasma bicarbonate concentrations were markedly decreased 10 minutes after exercise when compared to baseline and 120 minutes after exercise $(\mathrm{p}<0.01)$. Calcium in the plasma was significantly higher at 10 minutes after exercise $(\mathrm{p}<0.01)$, while it was even higher at 120 minutes post exercise $(\mathrm{p}<0.01)$. Plasma phosphorus was significantly lower when comparing baseline to 10 minutes post exercise $(\mathrm{p}<0.01)$ and recovered to 
Table 1. Serum chemistry mean and standard deviations before, 10 minutes after a 400 meter run and 120 minutes after exercise in 19 Greyhounds. ${ }^{a}$ indicates a significant difference between baseline racing samples $(\mathrm{p}<0.05)$. ${ }^{\mathrm{b}}$ indicates a significant difference between 10 minutes after racing and 120 minutes post racing $(\mathrm{p}<0.05)$.

\begin{tabular}{|c|c|c|c|}
\hline & 0 minutes & 10 minutes & 120 minutes \\
\hline Na (142 - 150 mEq/L) & $150.2 \pm 2.7$ & $152.7 \pm 2.9^{\mathrm{a}}$ & $146.3 \pm 3.5^{\mathrm{ab}}$ \\
\hline $\mathrm{K}(3.8-5.4 \mathrm{mEq} / \mathrm{L})$ & $5.4 \pm 0.3$ & $5.3 \pm 0.2$ & $4.5 \pm 0.4^{\mathrm{ab}}$ \\
\hline $\mathrm{Cl}(105$ - $116 \mathrm{mEq} / \mathrm{L})$ & $104.2 \pm 3.2$ & $99.6 \pm 2.4^{\mathrm{a}}$ & $104.2 \pm 2.8^{\mathrm{b}}$ \\
\hline Bicarbonate (mEq) & $20.1 \pm 2.3$ & $9.1 \pm 4.7^{\mathrm{a}}$ & $19.4 \pm 2.0^{\mathrm{b}}$ \\
\hline Ca (9.3 - 11.4 mg/dL) & $7.7 \pm 0.5$ & $9.0 \pm 1.4^{\mathrm{a}}$ & $10.1 \pm 0.6^{\mathrm{ab}}$ \\
\hline $\mathrm{P}(2.9$ - $5.2 \mathrm{mg} / \mathrm{dL})$ & $3.8 \pm 0.7$ & $2.6 \pm 0.4^{\mathrm{a}}$ & $3.6 \pm 0.5^{\mathrm{b}}$ \\
\hline $\mathrm{Mg}(1.4$ - $2.2 \mathrm{mEq} / \mathrm{L})$ & $1.8 \pm 0.3$ & $1.8 \pm 0.2$ & $1.7 \pm 0.1$ \\
\hline Total Protein (5.3 - 7 g/dL) & $6.1 \pm 0.8$ & $6.4 \pm 0.6$ & $5.5 \pm 0.3^{\mathrm{ab}}$ \\
\hline Globulin (1.9 - 3.6 g/dL) & $2.5 \pm 0.9$ & $2.3 \pm 0.4$ & $1.9 \pm 0.2^{\mathrm{ab}}$ \\
\hline Albumin (3.1 - $4.2 \mathrm{~g} / \mathrm{dL})$ & $3.6 \pm 0.3$ & $4.1 \pm 0.3^{\mathrm{a}}$ & $3.6 \pm 0.2^{\mathrm{b}}$ \\
\hline BUN (10 - 32 mg/dL) & $21.6 \pm 4.3$ & $21.9 \pm 4.2$ & $19.9 \pm 3.9^{\mathrm{ab}}$ \\
\hline Creatinine (0.6 - $1.4 \mathrm{mg} / \mathrm{dL})$ & $1.6 \pm 0.2$ & $1.7 \pm 0.2^{\mathrm{a}}$ & $1.7 \pm 0.2$ \\
\hline ALT (20 - 98 U/L) & $65.2 \pm 10.4$ & $80.5 \pm 16.5^{\mathrm{a}}$ & $68.2 \pm 11.7^{\mathrm{b}}$ \\
\hline AST (14 - 51 U/L) & $85.6 \pm 60.0$ & $76.4 \pm 35.7$ & $65.2 \pm 32.5^{b}$ \\
\hline ALP (17 - $111 \mathrm{U} / \mathrm{L})$ & $32.8 \pm 17.4$ & $43.6 \pm 17.6^{\mathrm{a}}$ & $37.7 \pm 12.6^{\mathrm{ab}}$ \\
\hline CK $(48-261 \mathrm{U} / \mathrm{L})$ & $484.1 \pm 394.6$ & $469.1 \pm 487.6$ & $347.6 \pm 432.6^{\mathrm{b}}$ \\
\hline
\end{tabular}

baseline concentrations by 120 minutes after exercise. Magnesium concentrations showed no significant difference across all three time points. Plasma osmolality was performed only before and 10 minutes after simulated racing and was significantly increased from $304.5 \pm 5.8$ to $317.5 \pm 13.7 \mathrm{mOsm} / \mathrm{L}(\mathrm{p}<0.01)$.

\subsection{Plasma Proteins, Urea and Creatinine}

Plasma total protein concentrations were modestly, but significantly decreased 120 minutes after exercise compared to 10 minutes after exercise $(\mathrm{p}<0.01)$ as well as baseline concentrations $(\mathrm{p}<0.01)$. The plasma globulins showed a similar pattern to total protein with the only significance being a difference at 120 minutes after exercise from the prior two blood draws $(\mathrm{p}=0.02)$. Plasma albumin had a significant rise 10 minutes after exercise ( $\mathrm{p}<0.01$ ) which returned to baseline within 120 minutes. Renal parameters were examined; plasma urea nitrogen showed no significant differences before and 10 minutes after exercise, however there was a significant decrease $(\mathrm{p}<0.01)$ between 120 minutes post exercise and prior time points examined. The plasma creatinine showed a mild significant increase 10 minutes after exercise $(\mathrm{p}<0.01)$ when compared to before or 120 minutes after exercise (Table 1 ).

\subsection{Plasma Enzymes}

Plasma liver enzymes revealed changes due to exercise (Table 1). ALT concentrations significantly increased 10 minutes after exercise $(\mathrm{p}<0.01)$ and returned to baseline within 120 minutes of exercise. Alkaline phosphatase activity was modestly increased from baseline $(\mathrm{p}<0.01) 10$ minutes after exercise and remained elevated at 120 minutes after exercise, but was mildly decreased from immediately after exercise $(p<0.01)$. Plasma AST and CK as markers of muscle cell permeability were not elevated from baseline 10 minutes after exercise and were significantly decreased from baseline by 120 minutes post exercise $(\mathrm{p}<0.01)$. 


\subsection{Plasma Insulin, Glucagon, NEFA and Glucose}

Plasma glucose concentrations were markedly increased 10 minutes after exercise $(81.7 \pm 27.3 \mathrm{mg} / \mathrm{dL})$, when compared to before exercise $(48.8 \pm 9.5 \mathrm{mg} / \mathrm{dL})$ and remained elevated at 120 minutes post exercise $(74.5 \pm 14.2$; p $<0.01$; Figure 1). The serum concentration of NEFA at baseline was $942.9 \pm 242.7$, while 10 minutes after exercise it was $244.0 \pm 94.2 \mathrm{mEq} / \mathrm{L}(\mathrm{p}<0.01)$ and returned to baseline at 120 minutes after exercise at $922.4 \pm$ $214.5 \mathrm{mEq} / \mathrm{L}$ (Figure 2). The serum insulin also increased from $7.1 \pm 1.7 \mathrm{uIU} / \mathrm{mL}$ at baseline to $10.8 \pm 7.2 \mathrm{uIU}$ $(p<0.03) 10$ minutes after racing, and returned below baseline at $4.0 \pm 2.4 \mathrm{uIU}$ by 120 minutes post exercise (p $<0.01$; Figure 3). The mean serum glucagon concentration at baseline was $68.1 \pm 24.0 \mathrm{pg} / \mathrm{mL}, 10$ minutes after exercise it was $63.3 \pm 20.3 \mathrm{pg} / \mathrm{mL}$, and 120 minutes after exercise was $58.9 \pm 20.8 \mathrm{pg} / \mathrm{mL}$ showing no significant difference between time points (Figure 4).

\subsection{Diet Analysis}

A daily portion of the diet for the average Greyhound was made containing 500 grams of ground whole chicken,

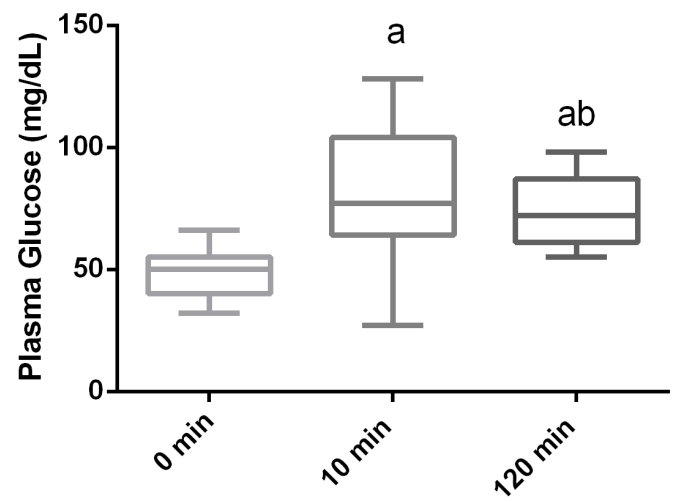

Figure 1. Box and whisker plots of plasma glucose concentrations immediately before 400 meter sprint and 10 minutes and 120 minutes after exercise in 19 Greyhounds. Boxes represent the median, $25^{\text {th }}$ and $75^{\text {th }}$ percentiles while the whiskers represent the minimums and maximums. a-represents a significant difference $(\mathrm{p}<0.05)$ from 0 minutes (resting). b-represents a significant difference $(p<0.05)$ from 10 minutes after exercise.

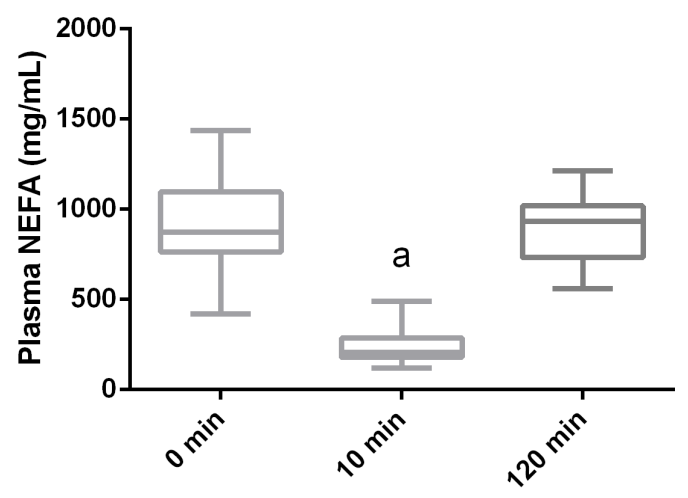

Figure 2. Box and whisker plots of plasma non-esterified fatty acids (NEFA) concentrations immediately before 400 meter sprint and 10 minutes and 120 minutes after exercise in 19 Greyhounds. Boxes represent the median, $25^{\text {th }}$ and $75^{\text {th }}$ percentiles while the whiskers represent the minimums and maximums. a-represents a significant difference $(\mathrm{p}<0.05)$ from 0 minutes (resting). 


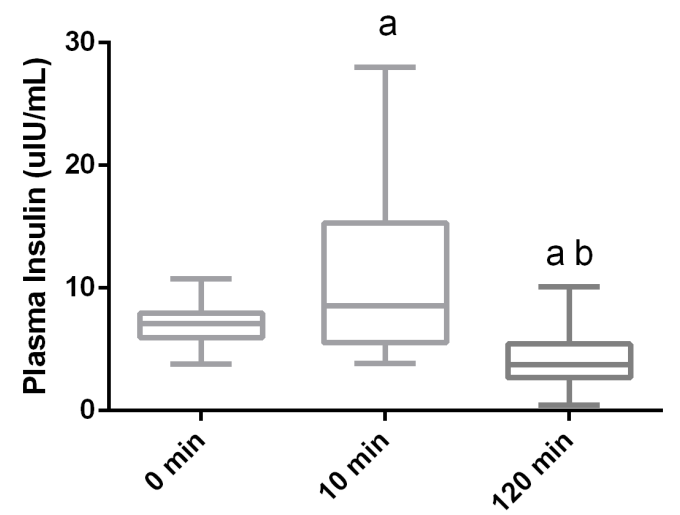

Figure 3. Box and whisker plots of plasma insulin concentrations immediately before 400 meter sprint and 10 minutes and 120 minutes after exercise in 19 Greyhounds. Boxes represent the median, $25^{\text {th }}$ and $75^{\text {th }}$ percentiles while the whiskers represent the minimums and maximums. a-represents a significant difference $(\mathrm{p}<0.05)$ from 0 minutes (resting). $\mathrm{b}-$ represents a significant difference $(p<0.05)$ from 10 minutes after exercise.

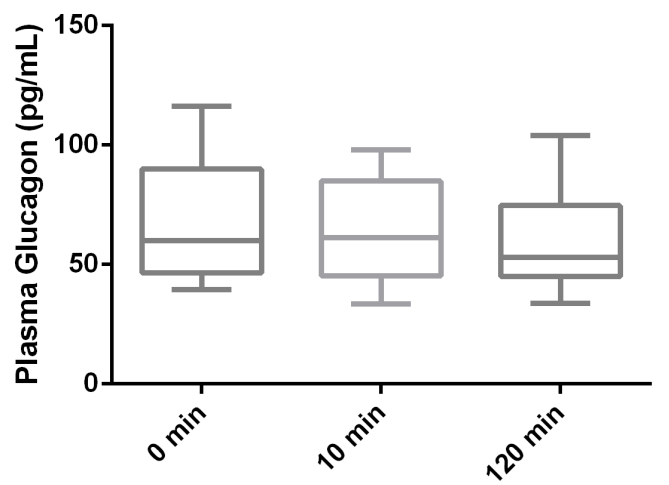

Figure 4. Box and whisker plots of plasma glucagon concentrations immediately before 400 meter sprint and 10 minutes and 120 minutes after exercise in 19 Greyhounds. Boxes represent the median, $25^{\text {th }}$ and $75^{\text {th }}$ percentiles while the whiskers represent the minimums and maximums.

500 grams of ground horse meat, 4 slices of whole wheat bread and 4 grams of Ranvet B Canine Multivitamin/Mineral supplement (Ranvet Corp, Banksmeadow NSW, Australia). The dietary constitution is reported in Table 2 based on dry matter and is adjusted for a typical $4000 \mathrm{kcal}$ diet. This adjustment was performed to allow for comparison to the National Research Council recommended daily intake based on dry matter content of a typical 4000 kilocalorie diet. Based on the analysis, this average daily ration contained approximately 2290 kilocalories and the metabolizable energy from protein, fat and carbohydrate were 42,41 and 17\%, respectively. When compared to the NRC recommended daily allowances of vitamins and minerals the diet was shown to be deficient in vitamin A, vitamin D, choline, sodium, iodine, selenium and copper (Table 2).

\section{Discussion}

Many of the serum biochemical alteration due to exercise were similar to those observed in other Greyhound studies [3] [5] [17] [19], showing rises in sodium and decreases in bicarbonate and chloride in the initial 10 minutes after exercise; while some studies found no alterations in chloride or potassium [4] [5]. The change in sodium is likely related to the increased osmolality post-exercise, while bicarbonate would be expected to fall as a compensatory mechanism for expected post-exercise lactic acidosis and rapid exchange of carbon dioxide due 
Table 2. Dietary analysis and calculation for the diet fed to the Greyhounds in this study compared to the National Research Council's recommended daily intake of essential nutrients based on a $4000 \mathrm{kcal}$ diet. Nutrient intake for the diet (4660 kcals/kg) was adjusted for the increased caloric content of the diet being fed. Vitamin $\mathrm{K}$ and biotin were not included due to gastrointestinal microbe contributions that cannot be accounted for.

\begin{tabular}{|c|c|c|}
\hline & Diet Dry Matter & NRC Dry Matter \\
\hline Protein (g) & 360.0 & 100 \\
\hline Fat (g) & 193.7 & 25 \\
\hline Carbohydrate (g) & 180.8 & NR \\
\hline Crude Fiber (g) & 4.1 & NR \\
\hline Calcium (g) & 15.3 & 4 \\
\hline Phosphorus (g) & 11.3 & 3 \\
\hline Magnesium (g) & 0.9 & 0.6 \\
\hline Sodium (mg) & 378.9 & 800 \\
\hline Potassium (g) & 6.3 & 4 \\
\hline Iron (mg) & 112.8 & 30 \\
\hline Zinc (mg) & 166.9 & 60 \\
\hline Copper (mg) & 4.2 & 6 \\
\hline Manganese (mg) & 20.8 & 4.8 \\
\hline Selenium (ug) & 64.8 & 350 \\
\hline Iodine (ug) & 333.3 & 880 \\
\hline Vitamin A (IU) & 3245.6 & 4545 \\
\hline Vitamin E (IU) & 72.1 & 30 \\
\hline Vitamin D (IU) & 433.0 & 552 \\
\hline Thaimine (mg) & 352.1 & 2.3 \\
\hline Niacin (mg) & 58.4 & 17 \\
\hline Riboflavin (mg) & 9.1 & 5.3 \\
\hline Pantothenate (mg) & 20.4 & 15 \\
\hline pyridoxine (mg) & 4.7 & 1.5 \\
\hline Folic acid (ug) & 436.8 & 270 \\
\hline Cobalamin (ug) & 34.9 & 35 \\
\hline Choline (mg) & 476.0 & 1,700 \\
\hline
\end{tabular}

$\mathrm{NR}$ - no requirements.

to panting [2] [19] [20]. Lactate concentrations were not recorded in this study, but were expected to rise precipitously due to the 400 meter sprint, creating some acidosis and a reciprocal decrease in plasma chloride observed. This accumulation of unmeasured anions, such as lactate, leads to a fall in the measured anions, chloride and bicarbonate, in order to maintain electroneutrality.

Within 120 minutes post-exercise sodium concentrations returned to below baseline, probably as a result of rehydration from post-exercise water consumption and re-establishment of intracellular and extracellular fluid homeostasis. Plasma potassium was no different after exercise, but was lower than baseline at the post $120 \mathrm{mi}-$ nutes most exercise time point. After exercise the potassium is expected to be elevated mildly due to hemoconcentration, however, the short 10 minute delay in blood collection after exercise may have been enough recovery time to allow for potassium normalization which is the time frame for normalization observed in other studies [5] [8]. 
Serum urea and creatinine changed little post-exercise, while others have shown more dramatic rises within the first 5 minutes after exercise [3] [5] [8]. Our less dramatic increase might be associated with plasma osmolality rise being approximately half of that observed in prior studies [5] [8]. Again, this might be a reflection the blood draw being 10 minutes after exercise allowing for skeletal muscle recovery and re-establishing of intracellular and extracellular fluid dynamics.

Contrary to other reports, our study showed that globulin concentrations decreased after exercise, especially by the 120 minutes mark [2] [6]. Globulin concentrations would have been expected to rise with the plasma fluid shifts, but did not. Similarly there were no rises in serum CK after exercise, yet the baseline concentrations were elevated in this group of dogs. We could not attribute this high CK to recent exercise, since the dogs were last exercised 3 days or prior to this simulated event. Resting CK values in other more recent studies in Greyhounds show values approximately half of what we observed [3] [4], suggesting some differences in kennel management of this particular population of Greyhounds. Although hemolysis can affect serum CK, the amount of hemolysis in a majority of the samples was minimal and should have had little to no significant effect [21]. In addition, serum phosphorus was depressed after exercise which is thought to be due to intracellular increases needed during glycolysis; however many studies have shown differing results which could be dietary relationships as well [3] [6] [8].

Further discrepancies in the blood work may be the result of dietary patterns, particularly due to the numerous deficiencies we observed in this diet. Prior field studies made no dietary assessment in relationship to the results reported [1] [5] [7] [8]. In this study, mineral deficits in copper, selenium and iodine were evident when compared with the National Research Council's (NRC) recommended daily intake [18]. The copper deficits were mild and likely insignificant since the copper content was around 70\% of NRC recommended daily intake which would be considered a minimum requirement; while the selenium deficit was more substantial leading to concerns related to antioxidant pathway sufficiency and might be related to the smoldering elevation in CK in these dogs [24]. Further examination of antioxidant status utilizing a diet of this nature is warranted on properly collected and stored serum or plasma samples. In addition, the inadequate dietary iodine intake has not been previously reported in the Greyhound literature; however low serum thyroid hormone concentrations in kennels of racing Greyhounds has been reported [22] [23]. In light of these findings, we speculate that some of the low serum thyroid concentration reported could be related to low iodine intake in populations of racing Greyhounds.

Insufficiency in dietary vitamin A and D were also noted, yet these concentrations in the in the diet were close to the presumed minimum adequate intake of these vitamins [25]. Choline intake was only around $25 \%$ of the adequate intake for dogs. Yet, choline hepatic synthesis can occur when dogs consume a high protein diet [25]. This diet supplied approximately 6 - 8 grams of protein per kilogram body weight which is 3 times the current NRC protein requirement, therefore hepatic choline synthesis might be adequate to make up for the low dietary intake. Equally concerning is the sodium deficiency in the diet. Although sodium is highly regulated through the renin-aldosterone angiotensin system, there is concern that the intake could result in clinical deficiency, yet plasma sodium was not perturbed in this group of Greyhounds. This is likely due to the fact that deficiencies noted when consuming $0.16 \mathrm{~g} / \mathrm{kg}$ dry matter or less in feed include obscure clinical signs such as polyuria, polydipsia, and increase heart rate. These dogs were consuming about twice that amount [25]. In addition, the higher kilocalorie consumption for daily energy requirements in Greyhounds leads to a $20 \%$ increase in consumption of all the mineral and vitamins intakes recommended [4], therefore many of the vitamin and mineral intakes might have been adequate due to the slightly higher energy intake of these dogs when compared with normal house dwelling domestic pet dogs.

The most striking glucoregulation difference observed in these dogs was the low resting blood glucose concentrations, compared to Greyhounds on higher carbohydrate commercial diets [3]-[5]. This lower resting plasma glucose concentration suggests some fundamental dietary differences. Hill and colleagues have shown that a high carbohydrate moderate protein diet may be beneficial to racing times in Greyhounds [3]. Greyhound trainers in Australia have fed diets similar to the one utilized in this study for many years, and in most cases have been reluctant to change despite the reported literature [26]. To our knowledge this is the first study to detect such a significant insulin rise post-exercise. Previous studies have reported that catecholamines increase during exercise and are responsible for regulation of hepatic glucose production [8] [10] [14]. Catecholamines would initially be expected to inhibit insulin-mediated glycogenesis [27] [28] [29], hence an insulin surge would not be expected until there was a catecholamine induced rise in glucose. This is likely the case as we observe an increase in glucose with a concomitant rise in plasma insulin concentrations 10 minutes post-exercise, with insulin 
being required to help transport the increased plasma glucose and free fatty acids intracellularly. This hyperglycemic-hyperinsulinemic response after glycogen-depleting exercise would create the appropriate milieu for restoration of muscle glycogen and energetics [1]. At 120 minutes after exercise there is a marked decrease in resting serum insulin concentrations to below baseline; most likely due to their not being fed at any time during that post-exercise interval.

Glucagon concentrations remained stable throughout the testing period. It is entirely possible that the bout of exercise was not long enough to stimulate the typical rise in glucagon for glycogenolysis or reciprocal decrease in the face of an insulin rise. Thirty minutes of modest exercise in sled dogs induces a rise in serum glucagon to promote glycogenolysis [30]. Our data suggest that the effects of glucagon in early regulation of hepatic glycogenolysis during short anaerobic activity are minimal to non-existent.

Non-esterified fatty acids have been reported to increase immediately after exercise [8], yet we showed a decrease in plasma NEFA at 10 minutes post-exercise. As previously mentioned, the catecholamine increase associated with strenuous exercise would have been expected to cause hepatic glycogenolysis and lipolysis leading to the release of glucose and free fatty acids into the circulation more rapidly than taken up by the working muscle. This could explain Snow et al.'s findings of elevated NEFA immediately post exercise [8]. The insulin surge that we detected 10 minutes post-exercise would then help to drive these fatty acids into the cells, hence explaining the subsequent fall in NEFA detected in this study.

Overall this work captures a distinct phase in elevated insulin concentrations which are responding to rises in serum glucose that are likely due to hepatic glycogenolysis which is expected after sprinting anaerobically. Surprisingly, glucagon is not responsive to short anaerobic activity. The plasma glucose at rest was lower than typical domestic dogs and increased significantly after exercise as a response to hepatic and muscle glycogenolysis associated with the acute bout of exercise and subsequent catecholamine surge. Ten minutes after exercise there is a pronounced decrease in non-esterified fatty acids, which may be due to insulin response in skeletal muscle promoting intracellular uptake for fuel. Serum chemistry parameters were typical for post exercise short anaerobic activity in Greyhounds except for a persistently elevated creatine kinase at all time points and decreases in plasma globulins well after recovery from exercise. The reasons for these differences observed are unknown; however, there were multiple subclinical deficiencies found in the raw meat-based diet formulation, including selenium which is involved in skeletal muscle integrity and antioxidant status.

\section{References}

[1] Dobson, G.P., Parkhouse, W.S., Weber, J.M., Stuttard, E., Harman, J., Snow, D.H. and Hochachka, P.W. (1988) Metabolic Changes in Skeletal Muscle and Blood of Greyhounds during 800-m Track Sprint. American Journal of Physiology-Regulatory, Integrative and Comparative Physiology, 255, R513-R519.

[2] Hill, R., Lewis, D., Randell, S., Scott, K., Omori, M., Sundstrom, D. and Butterwick, R. (2005) Effect of Mild Restriction of Food Intake on the Speed of Racing Greyhounds. American Journal of Veterinary Research, 66, 1065-1070. http://dx.doi.org/10.2460/ajvr.2005.66.1065

[3] Hill, R., Butterwick, R., Lewis, D., Scott, K., Omori, M., Jackson, M. and Doyle, C. (2001) Effect of Increased Dietary Protein and Decreased Dietary Carbohydrate on Performance and Body Composition in Racing Greyhounds. American Journal of Veterinary Research, 62, 440-447. http://dx.doi.org/10.2460/ajvr.2001.62.440

[4] Hill, R., Bloomberg, M., Legrand-Defretin, V., Burger, I., Hillock, S., Sundstrom, D. and Jones, G. (2000) Maintenance Energy Requirements and the Effect of Diet on Performance of Racing Greyhounds. American Journal of Veterinary Research, 61, 1566-1573. http://dx.doi.org/10.2460/ajvr.2000.61.1566

[5] Ilkiw, J., Davis, P. and Church, D. (1989) Hematologic, Biochemical, Blood-Gas, and Acid-Base Values in Greyhounds before and After Exercise. American Journal of Veterinary Research, 50, 583-586.

[6] Pieschl, R.L., Toll, P.W., Leith, D.E., Peterson, L.J. and Fedde, M.R. (1992) Acid-Base Changes in the Running Greyhound: Contributing Variables. Journal of Applied Physiology, 73, 2297-2304.

[7] Rose, R. and Bloomberg, M. (1989) Responses to Sprint Exercise in the Greyhound-Effects on Hematology, Serum Biochemistry and Muscle Metabolites. Research in Veterinary Science, 47, 212-218.

[8] Snow, D.H., Harris, R.C. and Stuttard, E. (1988) Changes in Haematology and Plasma Biochemistry during Maximal Exercise in Greyhounds. The Veterinary Record, 123, 487-491. http://dx.doi.org/10.1136/vr.123.19.487

[9] Stewart, I.B., Warburton, D.E.R., Hodges, A.N.H. and McKenzie, D.C. (2002) Splenic Contraction, Catecholamine Release, and Blood Volume Redistribution during Exercise in Man. Medicine \& Science in Sports \& Exercise, 34, S20. http://dx.doi.org/10.1097/00005768-200205001-00110 
[10] Miles, P.D., Finegood, D.T., Lickley, H.L. and Vranic, M. (1992) Regulation of Glucose Turnover at the Onset of Exercise in the Dog. Journal of Applied Physiology, 72, 2487-2494.

[11] Davenport, G.M., Kelley, R.L., Altom, E.K. and Lepine, A.J. (2001) Effect of Diet on Hunting Performance of English Pointers. Veterinary Therapeutics, 2, 10-21.

[12] McClelland, G., Zwingelstein, G., Taylor, C.R. and Weber, M. (1994) Increased Capacity for Circulatory Fatty Acid Transport in a Highly Aerobic Mammal. American Journal of Physiology-Regulatory, Integrative and Comparative Physiology, 266, 1280-1286.

[13] McClelland, G., Zwingelstein, G., Taylor, C. and Weber, J. (1995) Effect of Exercise on the Plasma Nonesterified Fatty-Acid Composition of Dogs and Goats: Species with Different Aerobic Capacities and Diets. Lipids, 30, 147-153. http://dx.doi.org/10.1007/BF02538268

[14] Marliss, E.B., Simantirakis, E., Miles, P.D., Purdon, C., Gougeon, R., Field, C.J. and Vranic, M. (1991) Glucoregulatory and Hormonal Responses to Repeated Bouts of Intense Exercise in Normal Male Subjects. Journal of Applied Physiology, 71, 924-933.

[15] Angle, C.T., Wakshlag, J.J., Gillette, R., Stokol, T., Geske, S. and Gregor, C. (2009) Haematologic, Serum Biochemical, and Cortisol Changes Associated with Anticipation of Exercise and Short Duration High-Intensity in Sled Dogs. Veterinary Clinical Pathology, 38, 370-374. http://dx.doi.org/10.1111/j.1939-165X.2009.00122.x

[16] Ermon, V., Yazwinski, M., Milizio, J.G. and Wakshlag, J.J. (2014) Serum Chemistry and Electrolyte Alterations in Sled Dogs before and after a 1600 km Race: Dietary Sodium and Hyponatraemia. Journal of Nutritional Sciences, 3, e26, 1-5

[17] Mawby, D.I., Bartges, J.W., d’Avignon, A, Laflamme, D.P., et al. (2004) Comparison of Various Methods for Estimating Body Fat in Dogs. Journal of American Animal Hospital Association, 40, 109-114. http://dx.doi.org/10.5326/0400109

[18] Beitz, D.C. (2006) Adult Dog Nutrient Requirement Appendices. In: Beitz, D.C., Ed., The National Research Council Dietary Nutrient Requirements of Dogs and Cats. 3rd Edition, National Academy Press, Washington DC, 357.

[19] Nold, J.L, Peterson, L.J. and Fedde, M.R. (1991) Physiological Changes in the Running Greyhound (Canisdomesticus): Influence of Race Length. Comparative Biochemistry and Physiological, 100A, 623-627. http://dx.doi.org/10.1016/0300-9629(91)90380-U

[20] Costill, D.L., Coyle, E., Dalsky, G., Evans, W., Fink, W. and Hoopes, D. (1977) Effects of Elevated Plasma FFA and Insulin on Muscle Glycogen Usage during Exercise. Journal of Applied Physiology, 43, 695-699.

[21] Koseoglu, M., Hur, A., Atay, A. and Cuhadar, S. (2011) Effects of Hemolysis Interferences on Routine Biochemistry Parameters. Biochemical Medicine (Zagreb), 21, 79-85. http://dx.doi.org/10.11613/BM.2011.015

[22] Gaughan K.R. and Bruyette D.S. (2001) Thyroid Function Testing in Greyhounds. American Journal of Veterinary Research, 62, 1130-1133.

[23] Shiel, R.E., Brennan, S.F., Omodo-Eluk, A.J. and Mooney, C.T. (2007) Thyroid Hormone Concentrations in Young, Healthy, Pretraining Greyhounds. The Veterinary Record, 161, 616-619.

[24] Kallfelz, F.A. (2006) Minerals. In: Beitz, D.C., Ed., The National Research Council Nutrient Requirements of Dogs and Cats, 3rd Edition, National Academy Press, Washington DC, 145-192.

[25] Morris, J.G. (2006) Vitamins. In: Beitz, D.C., Ed., The National Research Council Nutrient Requirements of Dogs and Cats, 3rd Edition, National Academy Press, Washington DC, 193-247.

[26] Kochane, E. (1998) Chapter 38: Management and Feeding Greyhounds. In: Bloomburg, M.S., Dee, J.F. and Taylor, R.A., Eds., Canine Sports Medicine and Surgery, Taylor, Philadelphia, 328-335.

[27] Macdonald, I.A., Bennett, T. and Fellows, I.W. (1985) Catecholamines and the Control of Metabolism in Man. Clinical Sciences, 68, 613-619.

[28] Raz, I., Katz, A. and Spencer, M.K. (1991) Epinephrine Inhibits Insulin-Mediated Glycogenesis but Enhances Glycolysis in Human Skeletal Muscle. American Journal of Physiology, 260, E430-E435.

[29] Laurent, D., Petersen, K.F. and Russell, R.R. (1998) Effects of Epinephrine on Muscle Glycogenolysis and Insulin-Stimulated Muscle Glycogen Synthesis in Humans. American Journal of Physiology, 274, E130-E138.

[30] Wakshlag, J.J., Snedden, K.A., Otis, A.M., Kennedy, C.A., Kennett, T.P., Scarlett, J.M., Kallfelz, F.A., Davenport, G.M., Reynolds, A.J. and Reinhart, G.A. (2002) Effects of Post-Exercise Supplements on Glycogen Repletion in Skeletal Muscle. Veterinary Therapeutics, 3, 226-234. 